\title{
Environmental coupling in ecosystems: From oscillation quenching to rhythmogenesis
}

\author{
Ramesh Arumugam and Partha Sharathi Dutte* \\ Department of Mathematics, Indian Institute of Technology Ropar, Punjab-140 001, India. \\ Tanmoy Banerję甲 \\ Department of Physics, University of Burdwan, West Bengal-713 104, India.
}

(Dated: September 4, 2018; Received :to be included by reviewer)

\begin{abstract}
How do landscape fragmentation affects ecosystems diversity and stability is an important and complex question in ecology with no simple answer, as spatially separated habitats where species live are highly dynamic rather than just static. Taking into account the species dispersal among nearby connected habitats (or patches) through a common dynamic environment, we model the consumer-resource interactions with ring type coupled network. Characterizing the dynamics of consumer-resource interactions in a coupled ecological system with three fundamental mechanisms: such as the interaction within the patch, the interaction between the patch and the interaction through a common dynamic environment, we report the occurrence of various atypical collective behaviors. We show that, the interplay between dynamic environment and dispersal among connected patches exhibits the mechanism of generation of oscillations, i.e., rhythmogenesis, as well as suppression of oscillations, i.e., amplitude death and oscillation death. Also, the transition of homogeneous steady state to inhomogeneous steady state occurs through a co-dimension-2 bifurcation. Emphasizing a network of spatially extended system, the coupled model exposes the collective behavior of synchrony-stability relationship with various synchronization occurrences such as in-phase and out-of-phase.
\end{abstract}

PACS numbers: 05.45.Xt, 87.23.Cc, 89.75.Fb

\section{INTRODUCTION}

For increasing complexity in ecosystems, modeling ecological consequences in continuously changing environmental conditions is one of the central concerns of theoretical ecologists. Various key components like environmental heterogeneity, habitat fragmentation, habitat loss and seasonal pattern or climatic change profoundly impact many biological phenomena such as synchrony of oscillating populations, community structure, diversitystability and synchrony-stability relationship [1 3]. In particular, habitat connectivity through species dispersal among fragmented landscapes play significant role in determining ecosystem functioning and evolutionary processes [4 [6]. Moreover, in spatial ecology, the connectivity of habitats conserves the ecological system by balancing the natural conditions 7]. As population movement prevents local species loss from complete extinction in their local habitat, so it is important to understand the factors which influences the dispersal effect. Numerous coupled nonlinear systems and coupled stochastic oscillators associated with dispersal conceptualize various biological notions starting from oscillations to chaotic behavior [8-10]. Particularly, in the context of species population dynamics, spatially extended dynamical systems with environmental heterogeneity play leading role in determining the community structure and in maintaining the biodiversity in regional landscapes [2, 11, 12].

\footnotetext{
* Corresponding author; parthasharathi@iitrpr.ac.in

$\dagger$ tbanerjee@phys.buruniv.ac.in
}

Much less is still known about the effects of species dispersal in dynamic habitats of ecosystems. Recently, in physical generic oscillators, dynamic environment has been considered in a scheme of indirect coupling to show various synchronization behavior [13] as well as synchronization of chaotic systems [14], amplitude death and oscillation death [15 17]. As far as ecological systems are concerned, in general, habitats are highly dynamic rather than static [7, 12] where species dispersal takes place in order to maintain the species diversity and persistence [18]. In search of preferential food, sometimes species move a long distance for suitable habitats [19]. While moving from one habitat to another habitat, species get the available food from the environment for their survival. Considering the varying environmental conditions as a dynamic variable, we model the consumer-resource interaction in a dynamic environment along with the presence of dispersion. We consider ecological oscillators represented by the Rosenzweig-MacArthur model under the simultaneous influence of two different types of couplings: One is the direct coupling describing the dispersal of species between spatially separated patches and another one is the indirect coupling describing a common dynamic environment for the patches which are connected via dispersal. As far as ecological environment is concerned, we consider the logistic growth model replicating a dynamic landscape. Our main concern in this paper is to investigate how the interplay of these two separate types of coupling affects the collective dynamical behavior of an ecological system.

In general, the concepts of nonlinear dynamics have been widely used in many studies on complex ecological systems to characterize various natural processes and 
its ecological perspectives [20, 21]. Emphasizing that, in coupled nonlinear systems, rhythmogenesis is an interesting dynamical phenomenon, such that the interaction through underlying coupling generates the oscillation from their respective steady states. Generation of rhythms from steady state is a novel phenomenon in most of the coupled physical, chemical and biological systems 22 24]. Especially, for regulation and restoration of oscillatory behaviors in various physiological and neuronal systems, rhythmogenesis is utilized as an important consequence of coupled dynamical systems in which the underlying coupling acts as a feedback factor [25 27] and drives the system away from steady state to oscillations. In contrast to the generation of oscillation from steady state, specific coupling involved in the system can drive coupled oscillators to oscillation quenching states, such as oscillation death (OD) and amplitude death (AD). In coupled oscillators, oscillation death (OD) is created by suppression of oscillations with formation of stable inhomogeneous steady states whereas AD is created by the suppression of oscillations with formation of stable homogeneous steady states [28, 29]. As far as ecological models are concerned, oscillation quenching mechanisms emphasize the relationship between dispersal induced stability and dispersal induced synchrony. Using mean-field coupling as species dispersal in spatially extended ecological model, the relationship between synchrony and stability induced by dispersal has been shown in Ref. [9, 10, 30].

In this paper, through a rigorous bifurcation analysis we show that environmentally coupled ecological systems exhibit both generation and suppression of oscillations, which are two diametrically opposite phenomena. Using this environmentally coupled model in homogeneous patches (i.e., identical patches), we show amplitude death, oscillation death, formation of inhomogeneous limit cycles and transition of $\mathrm{AD}$ to $\mathrm{OD}$ through a co-dimension-2 bifurcation. In particular, we emphasize that rhythmogenesis can occur along with different synchronized behavior. Although, the uncoupled RM model has simple characteristics, but due to presence of environmental coupling and dispersal among the fragmented habitats, the system exhibits many interesting dynamics which can be useful in the context of ecological systems. Moreover, we show that the dynamics of the considered system are qualitatively valid for a network of patches connected by a common environment and the system shows rhythmogenesis, perfect synchrony, in-phase synchrony, out-of-phase synchrony and the formation of multi-cluster state for a network of nearby connected patches.

We organize the paper as: First, the environmentally coupled ecological model along with the dynamics of uncoupled system as well as the dynamics of environment are described in Sec. III. Based on this model, the effect of dispersal and it's dynamics are shown in Sec. IV] in two different ways. One is based on the assumption that uncoupled patch is in oscillatory state and second one is started with steady state in uncoupled patch. Further, we extend to a network of ring coupled oscillators and robustness of this model is shown in Sec. V] Finally, we discuss the results from dynamical systems point of view and also its ecological interpretation in Sec. VI.

\section{MATHEMATICAL MODEL}

Taking into account the dispersal between two spatially separated patches in a dynamic environment, we model the consumer-resource interaction in a single system emphasizing the dynamics of consumer interaction in three ways. First one is the consumer interaction within the patch, second is consumers interaction between the connected patches through dispersal, and finally, the consumer interaction through a common dynamic environment. We consider, dynamics of the consumer $(H)$ and the resource $(V)$ interaction within a patchy habitat is given by the Rosenzweig-MacArthur (RM) model [21, 31]. Now, for the interaction between the isolated patches, we consider dispersal of consumer populations between spatially isolated patches. In fact, immigration and emigration are set by directly coupling the patches with dispersal rate $(d)$. Finally, while dispersing between patches, the consumer interacts with environment and gets the available resource from the environment for their survival during dispersal. This is potentially important for long distance dispersal of species. To describe the interaction through the environment, we consider another resource $(E)$ as a common dynamic environment and uncoupled dynamics of the environment is given by the logistic growth model. Further, the interaction between consumers $\left(H_{1,2}\right)$ and environment is governed by a type-II functional response [21, 32]. The choice of this particular function is motivated by the fact that it represents a functional response for many consumers. The shape of the function is based on the idea that, at low resource densities, consumers spend most of their time on searching for resources, whereas at high resource densities, they spend most of their time on resource handling [32]. The dynamics of consumer $(H)$ and resource $(V)$ for patch-1 and patch-2 along with the common environment $(E)$ are modeled as:

$$
\begin{aligned}
& \frac{d V_{1,2}}{d t}=r V_{1,2}\left(1-\frac{V_{1,2}}{K}\right)-\frac{\alpha V_{1,2}}{V_{1,2}+B} H_{1,2} \\
& \frac{d H_{1,2}}{d t}=H_{1,2}\left(\beta \frac{\alpha V_{1,2}}{V_{1,2}+B}-m\right)+d\left(H_{2,1}-H_{1,2}\right) \\
& \quad+\gamma \frac{\epsilon E}{E+C} H_{1,2} \\
& \frac{d E}{d t}=r_{1} E\left(1-\frac{E}{K_{1}}\right)-\frac{\epsilon E}{E+C}\left(H_{1}+H_{2}\right)
\end{aligned}
$$

where $r$ is the growth rate of the resource $(V)$ with $K$ as its carrying capacity, $\alpha$ and $B$ represent the predation rate of the consumer $(H)$ and half saturation constant respectively. The predation efficiency and mortality rate 
of the consumer $(H)$ are respectively given by $\beta$ and $m$. The environmental resource $(E)$ acts as another resource consisting of logistic growth dynamics. In this case, the growth rate and the carrying capacity of the environment $(E)$ are given by $r_{1}$ and $K_{1}$, respectively. Further, $\epsilon$ and $\gamma$ represent the predation rate and the predation efficiency of the consumer $(H)$ due to available resource from the common environment. In predatory level, $C$ is half saturation constant of the environment $(E)$. The equations (1-)-(15) emphasize that we use direct coupling between the consumers from neighboring patches via the dispersal rate $d$ and indirect coupling through the predation rate $\epsilon$ of the consumer $(H)$.

In all the previous studies [13 17] the environment has been considered as an overdamped oscillator having no intrinsic dynamics other than zero steady state: the environment is modulated in a linear way in the presence of systems that are coupled with it. This is an oversimplified view of any environment and thus, the true essence of (nonlinear) dynamical environment was missing in those studies. As the environment plays a key role in determining the dynamics of the coupled system, therefore, it is of fundamental interest to study the effect of an environment that is represented with some realistic model. Here, not only we take a realistic environment (logistic growth model) but, apart from that, unlike the previous studies [14 17] we also consider the interaction among the environment and systems to be nonlinear.

Interestingly, it can be shown that the model of environment considered in [14 17] viz. $\frac{d E}{d t}=-\mathcal{K} E$, where $\mathcal{K}$ is the damping parameter, is a special case of the general model given in Eq. (17). When $\epsilon=0$, from Eq. (1) the per capita rate of change of environment is $\frac{1}{E} \frac{d E}{d t}=\frac{f(E)}{E}=r_{1}\left(1-\frac{E}{K_{1}}\right)$ and hence declines by the quantity $-r_{1} / K_{1}$ for each individual added in the population. Let us consider that we perturb $E^{*}$ by a small quantity $s$, so the perturbed density is $E^{*}+s$. Now $\frac{d E}{d t}=\frac{d\left(E^{*}+s\right)}{d t}=\frac{d E^{*}}{d t}+\frac{d s}{d t}=\frac{d s}{d t}=f\left(E^{*}+s\right)$ as $\frac{d E^{*}}{d t}=0$. Expanding the function $f\left(E^{*}+s\right)$ about $E^{*}$ and neglecting the higher order terms we get $f\left(E^{*}+s\right)=$ $f\left(E^{*}\right)+\left.s \frac{d f}{d E}\right|_{E^{*}}$. As $f\left(E^{*}\right)=0$ and $\left.\frac{d f}{d E}\right|_{E^{*}}=-r_{1}$, so the approximate form of $\frac{d E}{d t}$ a small distance away from the equilibrium point $E^{*}=K_{1}$ is $\frac{d s}{d t}=-r_{1} s$, where $r_{1}>0$. Hence, if the steady state of the environment is perturbed by a small amount by some external factors it will actually behave like an overdamped oscillator.

Thus, the environment we have considered here resembles the overdamped model for a smaller perturbation, but additionally, it also accommodates the effect of larger perturbation exerted by the systems. Therefore, the environment considered here, indeed, represents a general model of environment. In the following we will emphasize on the results that come due to this nonlinear environment, and which were not observed for the previously considered simplified overdamped model of environment.

\section{STABILITY ANALYSIS}

The uncoupled dynamics of the RM model (i.e., Eqs. (1a)-(1b) with $d=0$ and $\epsilon=0$ ) are discussed in [9, 31]. Here we briefly discuss it for the sake of clarity. The RM model has two trivial equilibrium points (i.e., $\left(V^{*}, H^{*}\right)=(0,0)$ and $\left.\left(V^{*}, H^{*}\right)=(K, 0)\right)$ and one non-trivial equilibrium point (i.e., $\left(V^{*}, H^{*}\right)=$ $\left.\left(\frac{m B}{\alpha \beta-m}, \frac{B r \beta(K(\alpha \beta-m)-B m)}{K(\alpha \beta-m)^{2}}\right)\right)$. This non-trivial equilibrium point is stable if $\frac{B}{K}<\frac{\alpha \beta-m}{\alpha \beta+m}$ for certain parameter values of $K$. However, for a range of $K$ value, the nontrivial equilibrium point changes it's stability and raises to stable limit cycle. Hence, the RM model is in either steady state or in oscillation state depending upon different parametric values.

Now, let us investigate the stability of the coupled system given by Eq. (11). The trivial equilibrium points of the environmentally coupled system (11) are given by $(0,0,0,0,0),(K, 0,0,0,0),(0,0, K, 0,0),\left(0,0,0,0, K_{1}\right)$, $\left(K, 0,0,0, K_{1}\right), \quad\left(0,0, K, 0, K_{1}\right), \quad\left(K, 0, K, 0, K_{1}\right)$, $\left(V^{o}, H^{o}, V^{o}, H^{o}, 0\right)$ and a non-trivial equilibrium point is given by $\left(V^{*}, H^{*}, V^{*}, H^{*}, E^{*}\right)$. As the expression of the non-trivial equilibrium point is quite cumbersome, here we don't give the expression. However, the expressions for $V^{o}$ and $H^{o}$ are given by:

$$
\begin{aligned}
V^{o} & =\frac{B m}{\alpha \beta-m}, \text { and } \\
H^{o} & =-\frac{B r \beta(B m+K m-K \alpha \beta)}{K(\alpha \beta-m)^{2}} .
\end{aligned}
$$

The Jacobian matrix $(\mathcal{J})$ at the non-trivial equilibrium point $\left(V^{*}, H^{*}, V^{*}, H^{*}, E^{*}\right)$ is given by:

$$
\left.\mathcal{J}\right|_{\left(V^{*}, H^{*}, V^{*}, H^{*}, E^{*}\right)}=\left[\begin{array}{ccccccc}
j_{11} & j_{12} & : & 0 & 0 & : & 0 \\
j_{21} & j_{22} & : & 0 & j_{24} & : & j_{25} \\
\cdots & \cdots & \cdots & \cdots & \cdots \\
0 & 0 & : & j_{11} & j_{12} & : & 0 \\
0 & j_{24} & : & j_{21} & j_{22} & : & j_{25} \\
\cdots & \cdots & \cdots & \cdots & & \cdots \\
0 & j_{52} & : & 0 & j_{52} & : & j_{55}
\end{array}\right],
$$

where

$$
\begin{aligned}
& j_{11}=r-\frac{2 r V^{*}}{K}-\frac{B \alpha H^{*}}{\left(B+V^{*}\right)^{2}}, \quad j_{12}=-\frac{\alpha V^{*}}{\left(B+V^{*}\right)} \\
& j_{21}=\frac{B \alpha \beta H^{*}}{\left(B+V^{*}\right)^{2}}, \quad j_{22}=-d-m+\frac{\alpha \beta V^{*}}{\left(B+V^{*}\right)}+\frac{\gamma \epsilon E^{*}}{\left(C+E^{*}\right)} \\
& j_{24}=d, \quad j_{25}=\frac{c \gamma \epsilon H^{*}}{\left(C+E^{*}\right)^{2}}, \quad j_{52}=\frac{\epsilon E^{*}}{\left(C+E^{*}\right)} \text { and } \\
& j_{55}=r_{1}-\frac{2 r_{1} E^{*}}{K_{1}}+\frac{2 C \epsilon H^{*}}{C+E^{*}} .
\end{aligned}
$$

In the Jacobian $\mathcal{J}$, we have block matrices which simplifies the method of finding eigenvalues and the corresponding eigenvalues are given by the expression:

$$
\lambda_{1,2}=\frac{1}{2}\left(j_{11}+j_{22}-j_{24} \mp \sqrt{4 j_{12} j_{21}+\left(j_{11}-j_{22}+j_{24}\right)^{2}}\right)
$$


(a)
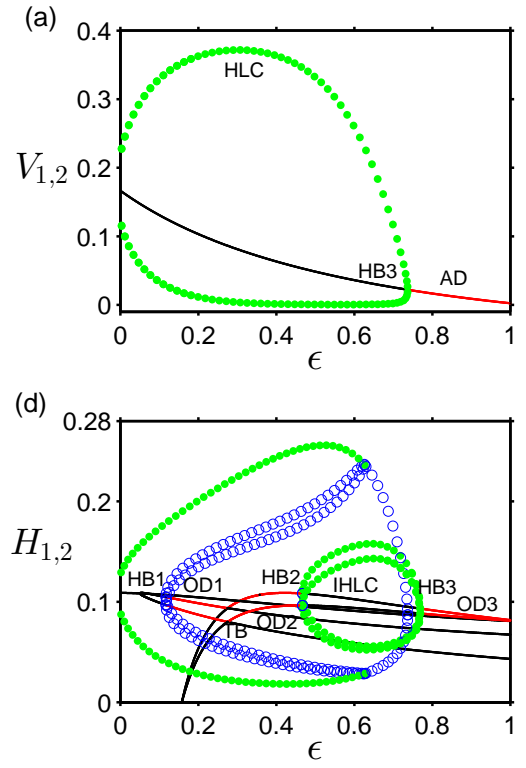

(b)

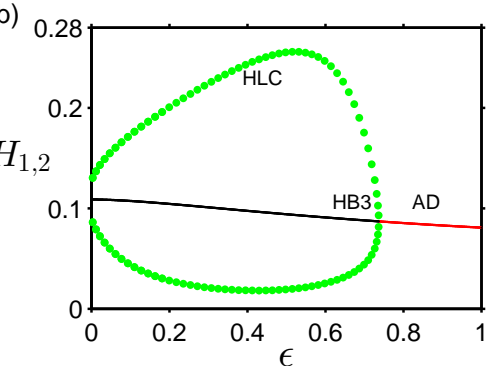

(e)

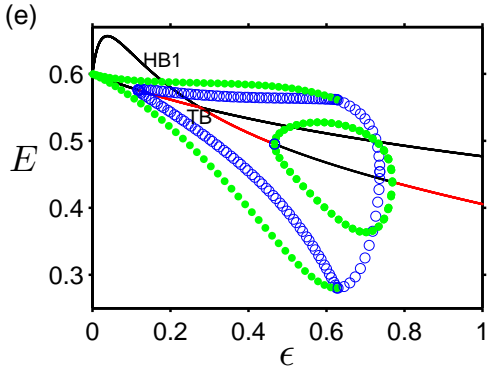

(c)

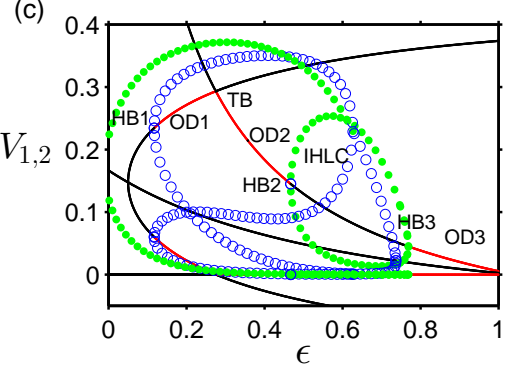

(f)

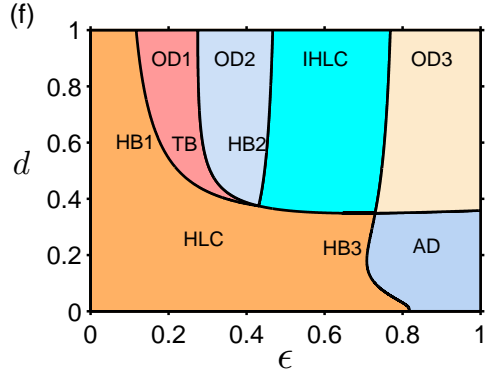

FIG. 1. (Color online) Oscillation and amplitude death: (a)-(b) One parameter bifurcation diagram for varying predation rate $(\epsilon)$ with lower value of dispersal rate $(d=0.2)$. (c)-(e) One parameter bifurcation diagram for varying predation rate $(\epsilon)$ with higher dispersal rate $(d=1)$. Red and black curves represent stable and unstable steady states respectively, whereas green and blue circles represent stable and unstable limit cycles respectively. Here OD, AD, HB, TB, IHLC and HLC represent oscillation death, amplitude death, Hopf bifurcation, transcritical bifurcation, inhomogeneous limit cycle and homogeneous limit cycle respectively. (f) Two parameter bifurcation diagram in $\epsilon-d$ plane. Other parameters are $r=0.5, K=0.5, \alpha=1, B=0.16$, $\beta=0.49, m=0.25, \gamma=0.6, r_{1}=0.5, K_{1}=0.6$ and $C=0.6$.

and $\lambda_{3,4,5}$ can be found out by the roots of the polynomial $x^{3}+a x^{2}+b x+c=0$, where

$$
\begin{aligned}
a= & -\left(j_{11}+j_{22}+j_{24}+j_{55}\right), \\
b= & -j_{12} j_{21}-2 j_{25} j_{52} \\
& +\left(j_{22}+j_{24}\right) j_{55}+j_{11}\left(j_{22}+j_{24}+j_{55}\right), \text { and } \\
c= & 2 j_{11} j_{25} j_{52}+j_{12} j_{21} j_{55}-j_{11} j_{55}\left(j_{22}+j_{25}\right) .
\end{aligned}
$$

From these eigenvalues, stability of the equilibrium point for different parameter values can be found. However, it has to be noted that due to the nonlinear nature of the coefficients of Jacobian, in most of the cases it is very difficult to predict the exact bifurcation points using eigenvalue analysis. Thus, to locate the bifurcation points and curves, we resort to the continuation package XPPAUT 33. The collective behavior of the coupled systems for different coupling and system parameters are described in the next section.

\section{RESULTS}

Emphasizing that, without coupling the RM model has either steady state or oscillatory state depending on the values of parameters, we qualitatively describe the coupled dynamics of system (1) in two distinct conditions: First, we consider the uncoupled patches are in oscillatory condition and examine how coupling affects the oscillation suppression states. Whereas, in the second case we consider the uncoupled patches are in equilibrium condition and investigate how coupling changes this quiescent state in order to establish any possible rhythmogenesis. In the numerical bifurcation analysis [33] we use the fourth-order Runge-Kutta algorithm with step size 0.001 .

\section{A. Uncoupled patches are in oscillatory state}

We start with the precondition that in the absence of dispersal (i.e., $d=0$ ) between the patch-1 and patch-2 and also the in the absence of coupling with environment (i.e., $\epsilon=0)$, the resource $(V)$ and the consumer $(H)$ show oscillations. Then, we study the simultaneous effects of dispersal and environmental coupling.

\section{Amplitude death in identical oscillators}

For lower dispersion rate determined by the parameter $d$, we observe that with increasing predation rate $\epsilon$, AD appears from stable limit cycle through an inverse supercritical Hopf bifurcation (HB3). Figures 1(a) and 1(b) show the $\mathrm{AD}$ state beyond $\epsilon_{H B 3}=0.7367$ for the resource $\left(V_{1,2}\right)$ and the consumer $\left(H_{1,2}\right)$, respectively for $d=0.2$. Here in both patch-1 and patch-2, populations are suppressed to homogeneous steady states. 

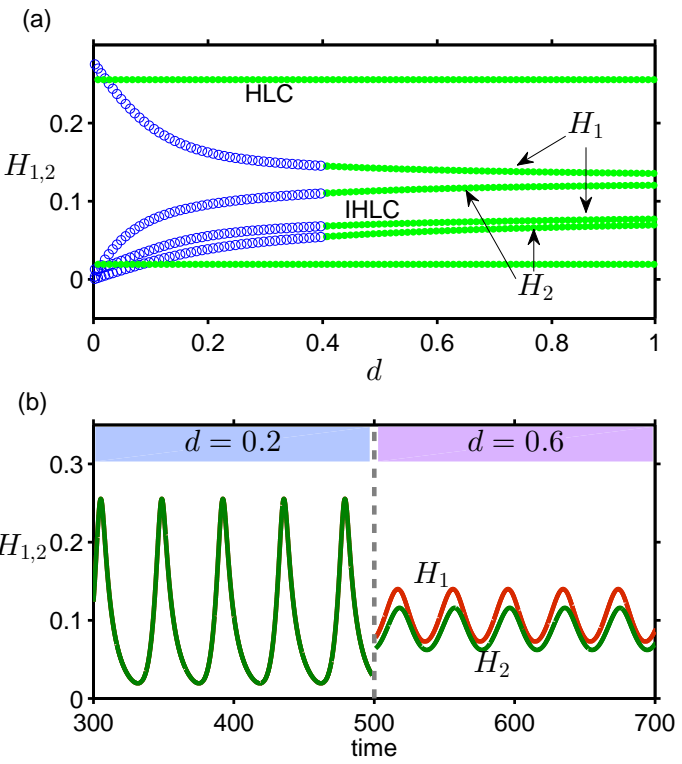

FIG. 2. (Color online) Transition from HLC to IHLC: (a) One parameter bifurcation diagram of $H_{1,2}$ with varying dispersal rate $d$. (b) Time series of $H_{1,2}$ which exhibits the direct transition from HLC to IHLC with change in $d$. We consider $\epsilon=0.5$ and all the other parameters are same as in Fig. 1

In Ref. [17] it has been shown that an environment modeled by an overdamped oscillator along with the linear modulation from the system can induce $\mathrm{AD}$ in the presence of dispersion in generic oscillators. But, the region of $\mathrm{AD}$ in the $d-\epsilon$ space was shown to be very narrow. In contrast to that, in our present case once $\mathrm{AD}$ occurs at $\epsilon_{H B 3}$ the system stuck to that state even for higher values of $\epsilon$. This contrast in results may be attributed to the fact that, unlike Ref. 17], here we consider a nonlinear environment and nonlinear coupling. For higher $d$, symmetry breaking is resulted, which is discussed below.

\section{Oscillation death for higher dispersal rate (d)}

Beyond a certain dispersal rate $d$, for increasing predation rate $(\epsilon)$, IHSS is created at $\epsilon_{P B}=0.05023$ : this IHSS gets stable and gives rise to oscillation death (OD1) at $\epsilon_{H B 1}=0.117$. Also, OD2 is created from OD1 through a transcritical bifurcation (TB) at $\epsilon_{T B}=0.2752$. It is noteworthy that, OD1 and OD2 are accompanied by a stable limit cycle, but OD3 that is created through supercritical Hopf bifurcation (HB3) for a higher dispersal value does not share the phase space with any oscillatory state. Moreover, for $m=0.25$, the dynamics of the environmental resource $(E)$ is shown in Fig. 1(e). Although, uncoupled dynamic environment $(E)$ is in steady state, it shows the synchronized oscillating behavior when its coupled with the oscillating patches.

In Fig. 1(c), OD1 shows inhomogeneous steady states

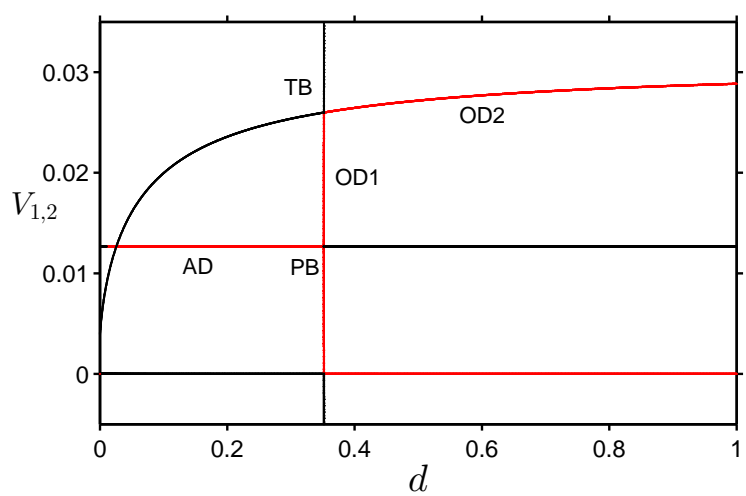

FIG. 3. (Color online) AD-OD transition: One parameter bifurcation diagram for varying $d$. Here PB represents Pitchfork bifurcation. Red and black curves represent stable and unstable steady states respectively. Other fixed parameters: $\epsilon=0.85, \alpha=1, r=0.5, K=0.5, B=0.16, \beta=0.49$, $r_{1}=0.5, K_{1}=0.6, C=0.6, \gamma=0.6$ and $m=0.25$

where $V_{1}, V_{2}, H_{1}, H_{2}$ and $E$ have non-zero density. But, in the OD2 state, the density of the resource $\left(V_{i}\right)$ in one patch is almost zero and another is non-zero. Surprisingly, in this situation, the consumers $\left(H_{1}\right.$ and $\left.H_{2}\right)$ from both patches have non-zero density both in OD1 and OD2 states. Even though the resource $\left(V_{i}\right)$ in one patch of OD2 is almost zero, but the consumer $\left(H_{i}\right)$ from that patch survives because of the available resource in the environment. Indeed, in the absence of resource $\left(V_{i}\right)$, the survival of consumer $\left(H_{i}\right)$ is completely supported by the environmental resource $(E)$ only.

The importance of direct coupling and environmental coupling and it's dynamics are shown for broader range of parameters using two parameter bifurcation diagram in $\epsilon-d$ space. Figure 1 (f) shows the parameter region where the oscillation quenching takes place in directly and indirectly coupled RM model. Each shaded region in Fig. 1(f) determines the dynamics of the coupled system for distinct values of $\epsilon$ and $d$. Moreover, when the dispersal rate $(d)$ is more than $d_{P B}=0.3519$ (approximately), OD1, OD2, IHLC and OD3 occur in the system, whereas only amplitude death occurs for low dispersal rate.

\section{Transition from $H L C$ to $I H L C$}

An interesting finding from Fig. प(f) is the transition from homogeneous limit cycle (HLC) to inhomogeneous limit cycles (IHLC) with the variation of dispersal rate $d$ for a certain range of $\epsilon$. The genesis of this transition can be understood more clearly from the one dimensional bifurcation diagram with $d$ for an exemplary value $\epsilon=0.5$. From Fig. 2(a) it is seen that for $\epsilon=0.5$ there exist HLCs even at $d=0$; additionally they are accompanied by unstable limit cycles [shown in open (blue) circles)]. As $d$ is increased, at $d_{H B}$ the unstable limit cycles get stable to give birth to IHLCs through subcritical Hopf bifurcation, 
(a)

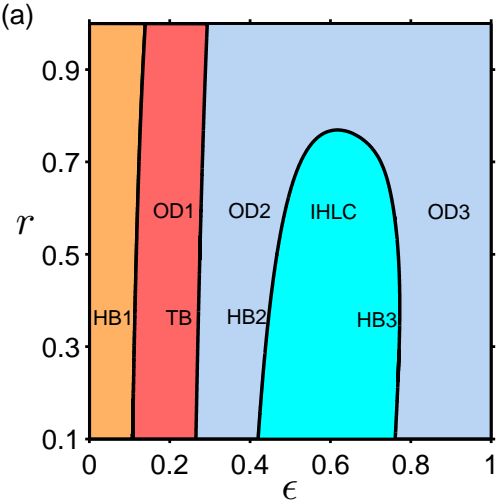

(b)

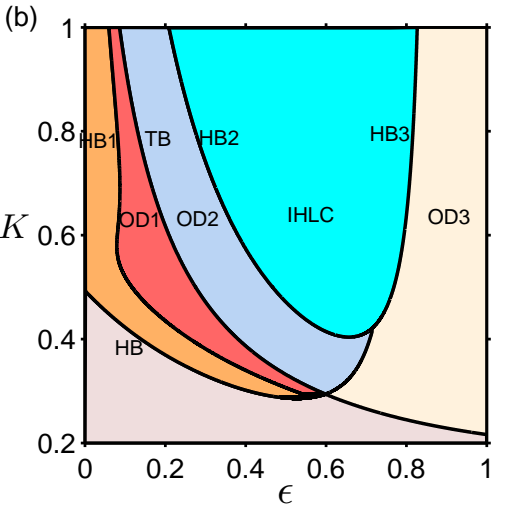

(c)

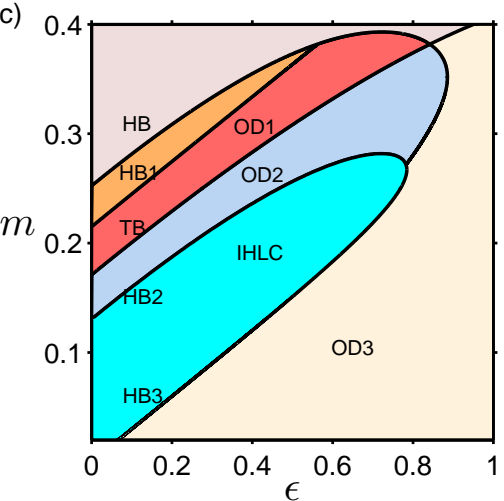

FIG. 4. (Color online) Local system parameters with environmental coupling parameters: (a) $\epsilon-d$ space as a two parameter bifurcation diagram, (b) $\epsilon-K$ space, (c) $\epsilon-m$ space. Here fixed parameters are $d=1, \alpha=1, B=0.16, \beta=0.49, r_{1}=0.5$, $K_{1}=0.6, C=0.6$ and $\gamma=0.6$. In each diagram, other fixed parameters are $r=0.5, K=0.5$ and $m=0.25$.

but the original HLCs remain there beyond that point. Thus, beyond $d_{H B}$ we can get a direct transition from HLC to IHLC for an appropriate choice of initial conditions. Figure 2(b) shows this transition in time series of HLC (at $d=0.2)$ and IHLC $(d=0.6)$ [we take $\epsilon=0.5$ ].

In the $\epsilon$ space (for a fixed $d$ ), it can be seen from Figs. 1(c) and 1(d), that inhomogeneous limit cycles (IHLC) are formed at $\epsilon_{H B 2}=0.467$ through a supercritical Hopf bifurcation (HB2) without considering any mismatch in species local dynamics. Further this IHLC are suppressed to inhomogeneous steady states which give raise to OD3.

\section{AD to $O D$ transition}

From Fig.1(f), it is interesting to note that for a higher $\epsilon$ value (organized by HB3 curve) the system shows either $\mathrm{AD}$ or OD depending upon the dispersal rate $(d)$. Thus, if we fix the value of $\epsilon$ in this oscillation suppressed region and vary $d$ then a continuous direct transition from $\mathrm{AD}$ to OD is observed. But, unlike other AD-OD transition, here the dynamics is governed by the co-dimension two bifurcation.

This is shown more clearly in Fig. 3, which shows the direct transition of $\mathrm{AD}$ to $\mathrm{OD}$ with the symmetry breaking of steady state through pitchfork bifurcation (PB). For low dispersal rate, system (11) shows $\mathrm{AD}$ whereas for increasing dispersal rate, OD1 occurs at $d_{P B}=0.3519$. Further an increase in dispersal $(d)$, OD2 is created through transcritical bifurcation (TB) at $d_{T B}=0.3519$ which is same as the pitchfork bifurcation point at $d_{P B}=0.3519$. This shows that $d=0.3519$ is a point of co-dimension 2 bifurcation. It is important to note that consumers from patch- 1 and patch-2 $\left(H_{1,2}\right)$ and also the resource $\left(V_{1,2}\right)$ are in non-zero density in OD1 state whereas the resource $\left(V_{1,2}\right)$ density in OD2 is in low value.

\section{Effect of local dynamics}

Relative to the coupling parameters (i.e., $d$ and $\epsilon$ ), other parameters representing behavioral, morphological and life history traits (characteristics) such as growth rate $(r)$, carrying capacity $(K)$ and mortality rate $(m)$ also significantly contribute in the dynamics of spatial ecosystems. The resource $(V)$ from both patch-1 and patch-2 doesn't involve in both direct and environmental coupling. However, it indirectly plays the role in determining the oscillation death and amplitude death. Here spatial variation is taken into account, we show the two parameter bifurcation diagram for varying the local parameters (i.e., $r, K$ and $m$ ) along with varying predation rate $(\epsilon)$.

In Fig. 4, two parameter bifurcation diagrams in $\epsilon-r$ plane, $\epsilon-K$ plane and $\epsilon-m$ plane are shown with different color shaded regions representing distinct dynamics of the system (11) for different parameters. Figures 1(a)-(e) depict one particular case of Fig. 4 with distinct change in each parameter. Here change in growth rate $(r)$ does not affect much in the OD1 region (shown in Fig. 4(a)) but changes the OD2 region by shrinking inhomogeneous limit cycles. In fact, for higher growth rate $(r)$, the inhomogeneous limit cycles vanish and further OD2 and OD3 coincides together and thus forms only one OD. Same conclusion holds for decreasing the carrying capacity $(K)$. For low value of the carrying capacity, no oscillation occurs (shown in Fig. 4(b)). As far as local dynamics of the patch is concerned, mortality rate $(m)$ is an important factor in heterogeneous fragmented landscapes. We check the dynamics for varying mortality rate and predation rate. For increasing mortality state, OD region and inhomogeneous limit cycles disappear (shown in Fig. 四(c)). At low mortality rate, IHLC and OD occur whereas for higher mortality rate, we have only homogeneous limit cycles along with oscillation death through HB3. Importantly, both environmental coupling parameters and local dynamical parameters determine the dynamics of the considered system. 
(a)
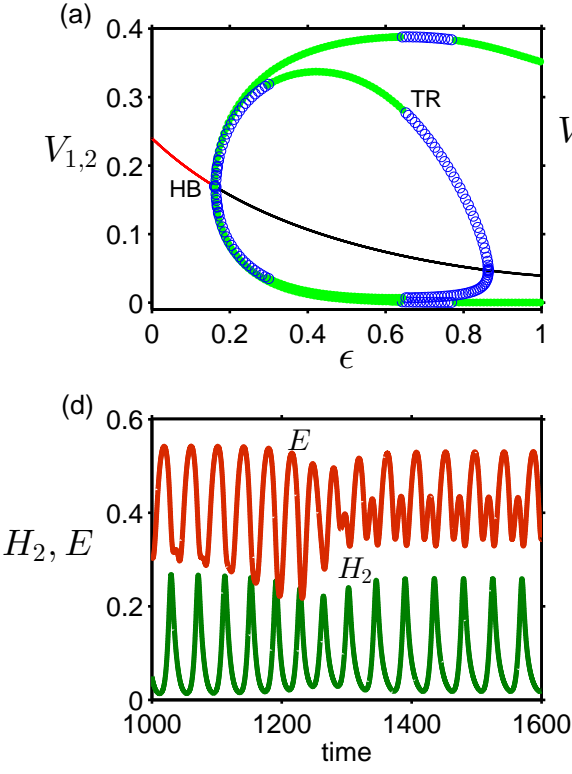

(b)

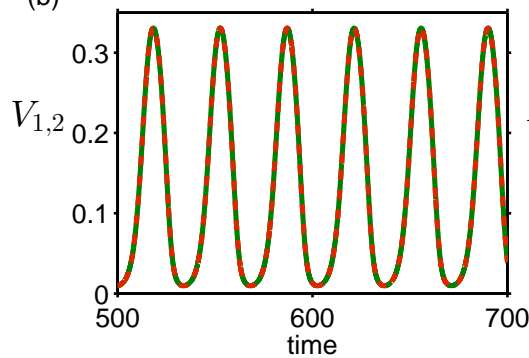

(e)

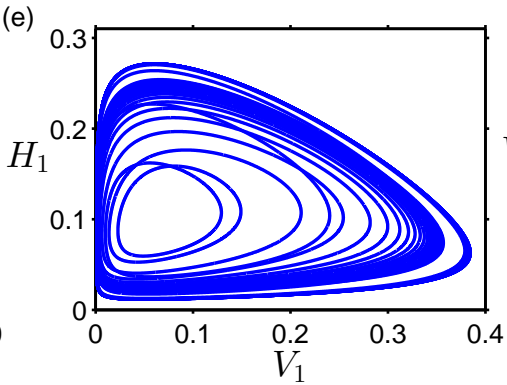

(c)

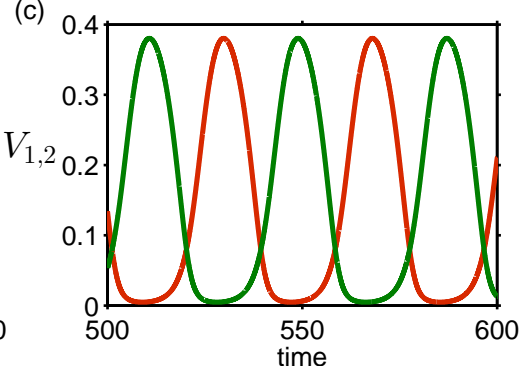

(f)

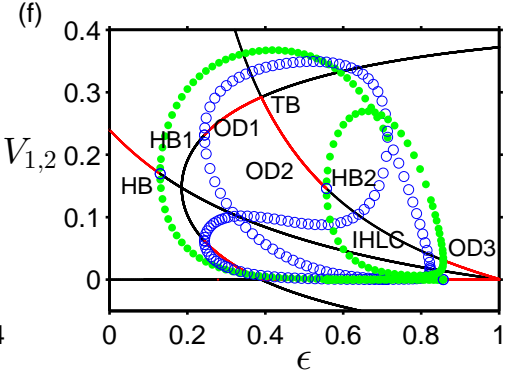

FIG. 5. (Color online) (a) One parameter bifurcation diagram for varying $\epsilon$ with $d=0$. TR represents Torus bifurcation. Other fixed parameters are $\alpha=1, r=0.5, K=0.5, B=0.16, \beta=0.5, r_{1}=0.5, K_{1}=0.6, C=0.5, \gamma=0.5$ and $m=0.3$. (b) Perfect synchrony for $\epsilon=0.5$ with the initial condition $\left(V_{1}, H_{1}, V_{2}, H_{2}, E\right)=(0.28,0.051,0.24,0.052,0.55)$. (c) Out-of-phase synchrony for $\epsilon=0.5$ with a different initial condition $\left(V_{1}, H_{1}, V_{2}, H_{2}, E\right)=(0.297,0.145,0.016,0.0308,0.5)$. (d) Time series of chaotic behavior for $\epsilon=0.76$ (after the Torus bifurcation (TR)). (e) Phase space of the chaotic time series. In (b)-(e), parameters are same as in (a). (f) One parameter bifurcation diagram for varying $\epsilon$ with fixed $d=1$. Here other parameters are $\alpha=1$, $r=0.5, K=0.5, B=0.16, \beta=0.5, r_{1}=0.5, K_{1}=1, C=0.5, \gamma=0.5$ and $m=0.3$.

\section{B. Uncoupled patches are in equilibrium state}

All the results shown in the previous section are based on the initial assumption that the dynamics of uncoupled system is in oscillatory state. Now we explore the effect of direct and indirect coupling when the uncoupled systems are in equilibrium state. We set the individual uncoupled systems in steady state by choosing proper value of mortality rate $(m)$ of the consumer $(H)$.

\section{Rhythmogenesis and oscillation death}

In this section, we set that each patch is in equilibrium state by choosing the mortality rate of the consumer as $m=0.3$. We check the dynamics of the system (10) in two different cases. One is absence of direct coupling and another is considered with direct coupling along with coupling through the environment.

\section{Case - I : Absence of direct coupling}

In the absence of direct coupling (i.e., $d=0$ ), we show the dynamics of system (11) using one parameter bifurcation diagram for varying $\epsilon$ with resource density in Fig. 5(a). Interestingly, oscillations are created at $\epsilon_{H B}=0.1605$ in the presence of environmental coupling alone through supercritical Hopf bifurcation. The gener- ation of oscillation through coupling is termed as rhythmogenesis in the literature [22, 23]. Hence, the presence of environmental coupling drives the steady state to oscillatory state and creates rhythmogenesis with finite period of oscillation. Moreover, torus bifurcation is created here with chaotic dynamics (shown in Fig. 5 (a)). The torus bifurcation is associated with the Neimark-Sacker bifurcation of the Poincaré map of a limit cycle in ordinary differential equation. Sometimes torus bifurcation is also referred as secondary Hopf bifurcation and occurs at the double-Hopf bifurcation of the equilibrium point in continuous dynamical system. In Fig. 5 (a), torus bifurcation occurs at $\epsilon_{T R}=0.652$ with a pair of eigenvalues $0.6907 \pm 0.7232 i$ which has unit modulus. We check back and forth of this Torus bifurcation for different $\epsilon$ value. When $\epsilon=0.6518$, a pair of Floquet multipliers $0.6905 \pm 0.7224 i$ exists with absolute value 0.9993 whereas when $\epsilon=0.6528$, a pair of Floquet multiplier $0.6917 \pm 0.7267 i$ exists with absolute value 1.003. Further, corresponding trajectories and phase space of chaotic dynamics are shown in Figs. 5(d) and 5(e).

As there is no dispersal (i.e., no direct coupling) between the patches, only environmental coupling plays role in determining the dynamics here. In Fig. 5 (a), it is shown that two limit cycles occur together with a torus bifurcation. The upper one is in out-of-phase synchrony, whereas lower one shows the perfect synchrony between the patches. In fact, in lower one, torus bifurcation 

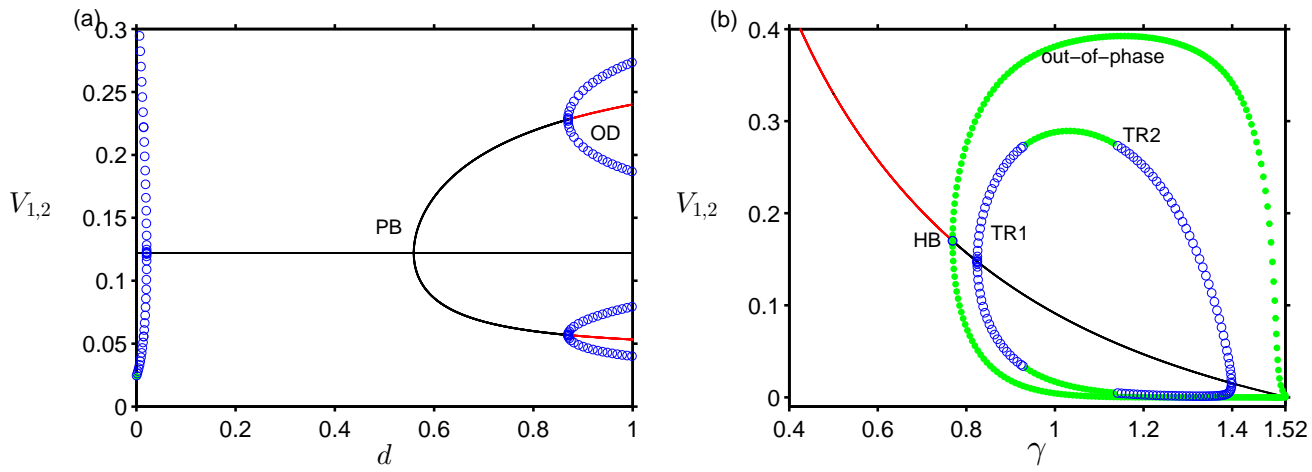

FIG. 6. (Color online) Direct coupling: (a) one parameter bifurcation diagram for varying $d$ with the fixed parameters $\epsilon=0.1636, \alpha=1, r=0.5, K=0.5, B=0.16, \beta=0.5, r_{1}=0.5, K_{1}=0.6, C=0.5, \gamma=0.5$ and $m=0.27$. (b) One parameter bifurcation diagram for varying predation efficiency $\gamma$ for fixed parameters $d=0, \alpha=0.6, r=0.5, K=0.5, B=0.16, \beta=0.5$, $r_{1}=0.5, K_{1}=0.7, C=0.3, \epsilon=0.3$ and $m=0.3$.
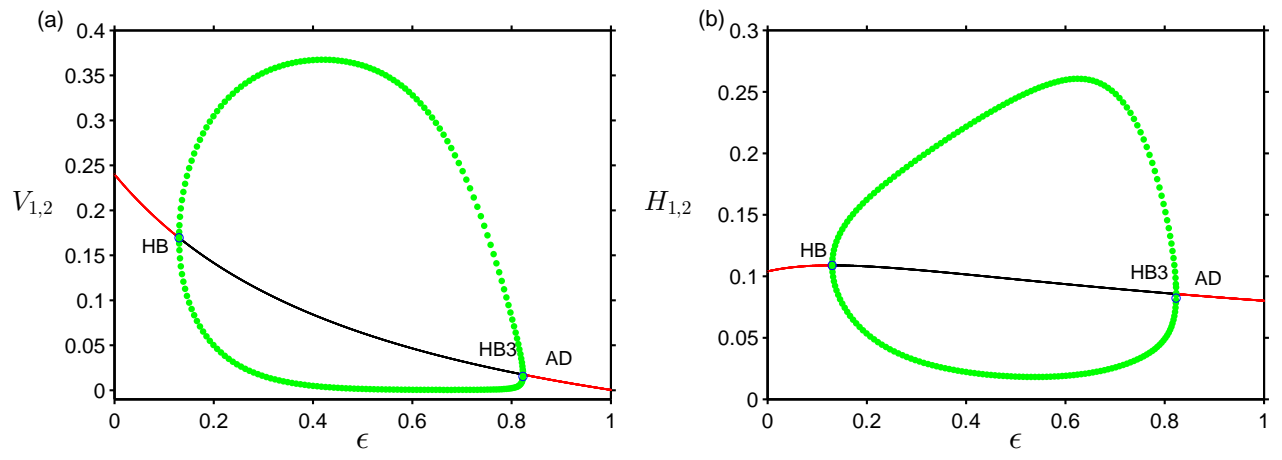

FIG. 7. (Color online) Amplitude Death :(a) -(b) One parameter bifurcation diagram for varying $\epsilon$ for the fixed parameters $\alpha=1, r=0.5, K=0.5, B=0.16, \beta=0.5, r_{1}=0.5, K_{1}=1, C=0.5$ and $m=0.3$. Other fixed parameters are $\gamma=0.5$ and low value in direct coupling strength $d=0.3$.

occurs. From this two limit cycles, depending on the initial conditions, we get either perfect synchrony or out-of-phase synchrony. Figures 5 (b) and 5 (c) show time series of perfect and out-of-phase synchrony of the resource $\left(V_{1,2}\right)$ for fixed $\epsilon=0.5$, but with different initial conditions. For increasing predation rate $(\epsilon)$, the system shows the chaotic behavior and perfect synchrony. At $\epsilon=0.76$, in Figs. 5(d) and 5(e) time series and phase space are shown respectively. Hence, perfect synchrony, out-of-phase synchrony and chaotic dynamics occur with presence of indirect coupling.

\section{Case - II : Presence of direct and indirect coupling}

With the presence of direct coupling (i.e., $d \neq 0$ ), occurrence of oscillation death is shown in Fig. $5(\mathrm{f})$ for increasing the predation rate $(\epsilon)$. For this case also, rhythmogenesis occurs through the variations in the coupling parameter $\epsilon$. In fact, coupling drives the steady state to oscillatory state which further quenched to oscillation death as mentioned in the earlier section. First oscillation are generated through supercritical Hopf bifurcation at $\epsilon_{H B}=0.1301$ and then OD1 is created at $\epsilon_{H B 1}=0.2438$ by symmetry breaking of steady state through pitchfork bifurcation. Further, OD2 is created through transcritical bifurcation at $\epsilon_{T B}=0.3899$. Moreover, inhomogeneous limit cycles arise at $\epsilon_{H B 2}=0.557$ which is further suppressed to OD3 at $\epsilon=0.857$ [shown in Fig. [5(f)].

For fixed direct coupling parameter $(d)$, we have shown the various dynamics of system (10) with variations in environmental coupling parameters. In contrast, for fixed environmental coupling parameter, we find the dynamics for varying direct coupling parameter. We show the one parameter bifurcation diagram for varying the coupling strength $(d)$ in Fig. 6(a). Important to note that here we always start with steady state in uncoupled patches. OD is created at $d_{O D}=0.8698$ by symmetry breaking of steady state through pitchfork bifurcation (PB).

\section{Perfect and out-of-phase synchrony}

As we use environmental coupling to connect the indirect interaction of the consumers between the patches via a common environment, parameters such as predation rate $(\epsilon)$ and predation efficiency $(\gamma)$ in the environ- 

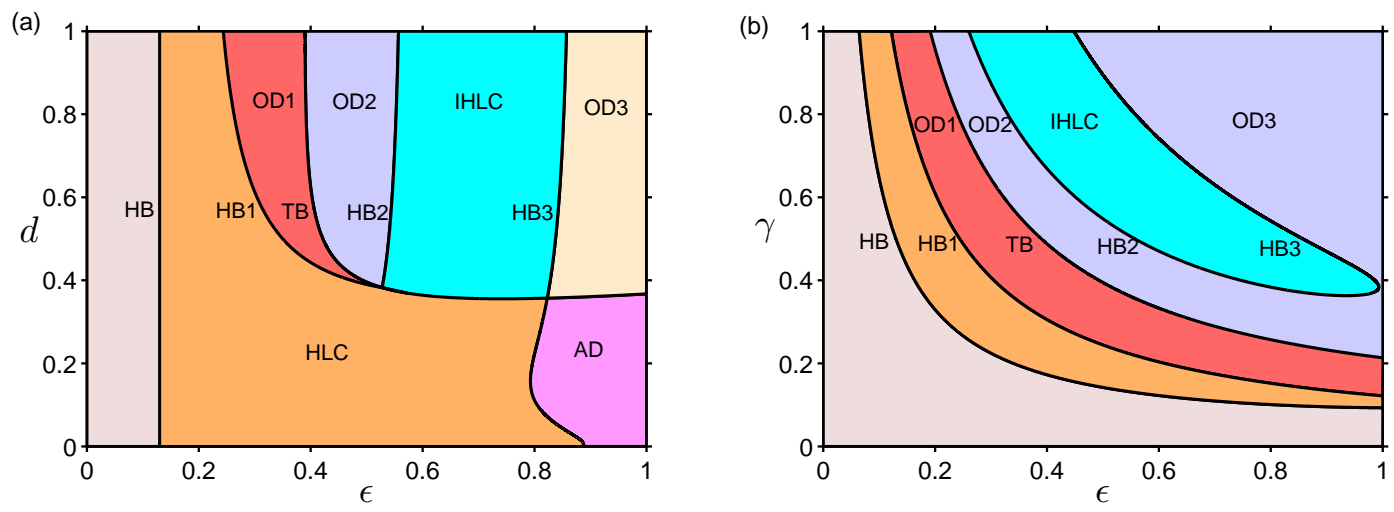

FIG. 8. (Color online) Two parameter bifurcation diagram: (a) $\epsilon-d$ space for fixed $\gamma=0.5$, (b) $\epsilon-\gamma$ space for fixed $d=1$. Other fixed parameters are $\alpha=1, r=0.5, K=0.5, B=0.16, \beta=0.5, r_{1}=0.5, K_{1}=1, C=0.5$ and $m=0.3$.

mental coupling determines the intrinsic dynamics of the system. So far, for changing predation rate $(\epsilon)$, we determine the dynamics of this system. However, for increasing predation efficiency $(\gamma)$, we show other interesting dynamics using the one parameter bifurcation diagram in Fig. 6(b). As mentioned earlier in Fig. 囵 here oscillations are created through coupling and further we have out-ofphase, in-phase synchrony and perfect synchrony among the patches. Also, we have torus bifurcation where the stable limit cycles transitioned to unstable limit cycles. For a particular $\gamma$ value, we have perfect and out-of-phase synchrony which occur completely depending on the initial conditions.

\section{Rhythmogenesis and amplitude Death $(A D)$}

As we start with fixed steady state in the uncoupled patch, in all the cases for an increase in predation rate, oscillations are created and further suppressed to inhomogeneous steady states. In another parametric set up and for low value of direct coupling strength $(d)$, we have rhythmogenesis (homogeneous limit cycles) at $\epsilon_{H B}=0.1301$ and suppressed steady states at $\epsilon=0.823$ occur for increasing predation date $(\epsilon)$. In this case, one parameter bifurcation diagrams for both consumer and resource are given in Figs. 7(a) and 7(b), respectively.

The robustness of the creation of oscillation and oscillation death is identified by $\epsilon-d$ space and $\epsilon-\gamma$ space in Figs. 8(a) and 8(b) using two parameter bifurcation diagram. For low to high dispersal rate $(d)$, rhythmogenesis always occurs at $\epsilon_{H B}=0.1301$ which is a limit point to oscillation creation. For low dispersal rate, rhythmogenesis and $\mathrm{AD}$ take place whereas for higher coupling strength, both rhythmogenesis, oscillation death and IHLC occur (shown in Fig. 8(a)). Also, for increasing predation efficiency in the environmental coupling, we have death state and inhomogeneous limit cycles. However, for higher predation rate and predation efficiency, only oscillation death (OD3) occurs (shown in
Fig. 8(b)).

\section{NETWORK STRUCTURE}

We extend the system (11) to a network consisting $N$ number of patches coupled by a common dynamic environment $(E)$ and also dispersal takes place among the connected patches. Using a ring type coupled network (i.e., we consider the periodic boundary condition), we set that each patch is connected to its nearest neighbor. So the dynamics of the consumer $(H)$ and the resource $(V)$ in the $i^{t h}$ patch along with common dynamic environment $(E)$ are given by:

$$
\begin{aligned}
\frac{d V_{i}}{d t}= & r V_{i}\left(1-\frac{V_{i}}{K}\right)-\frac{\alpha V_{i}}{V_{i}+B} H_{i} \\
\frac{d H_{i}}{d t}= & H_{i}\left(\beta \frac{\alpha V_{i}}{V_{i}+B}-m\right)+d\left(H_{i+1}-2 H_{i}+H_{i-1}\right) \\
& +\gamma \frac{\epsilon E}{E+C} H_{i}, \quad i=1,2, \ldots, N \\
\frac{d E}{d t}= & r_{1} E\left(1-\frac{E}{K_{1}}\right)-\sum_{i=1}^{N} \frac{\epsilon E}{E+C} H_{i}
\end{aligned}
$$

Since many patches are connected in network with a common environment, we consider a high carrying capacity $\left(K_{1}\right)$ of the environment so that it can support many connected patches.

We check the robustness of our results at different levels of dispersal rate for a network consisting 16 patches and a common dynamic environment. First for low values of $\epsilon$, Fig. 9(a) shows the synchronized oscillations of 16 patches when dispersal rate $d=0.35$. Time series of perfectly synchronized homogeneous limit cycles (HLC) of the consumer $(H)$ is shown in Fig. 9(f).

With an increase in $\epsilon$ and choosing appropriate initial conditions, Fig. 9(b) shows inhomogeneous limit cycles for the same dispersal rate $d=0.35$. Corresponding trajectories are shown in Fig. 9(g). From this, it is clear 


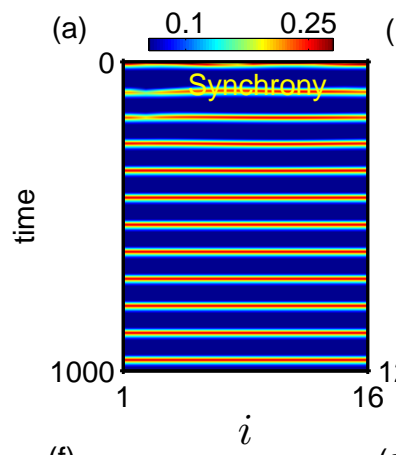

(f)

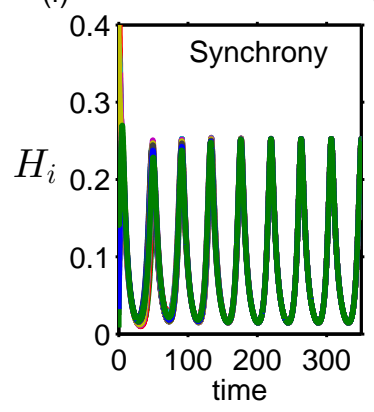

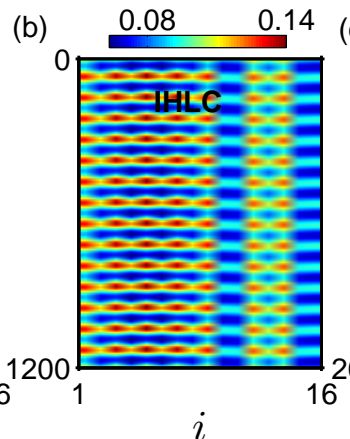

(g)

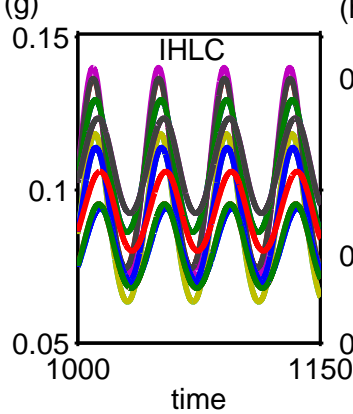

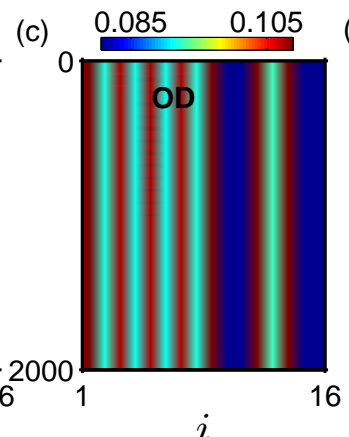

(h)

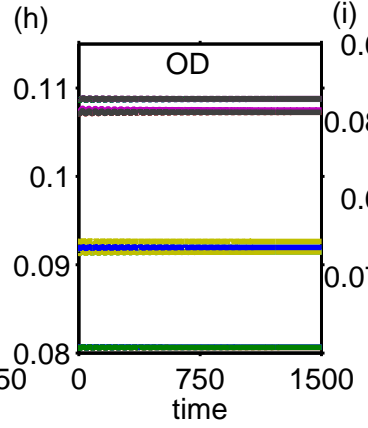

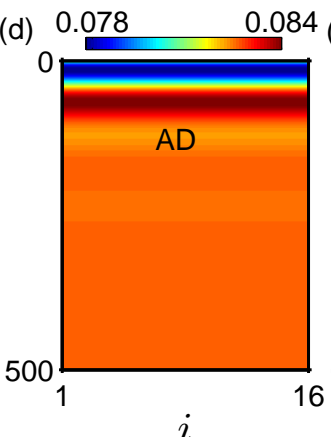

(i)

(i)

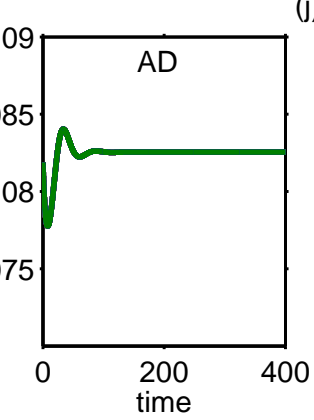

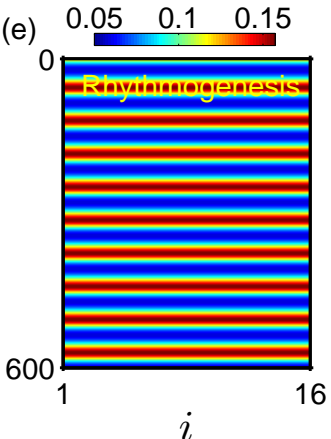

(j)

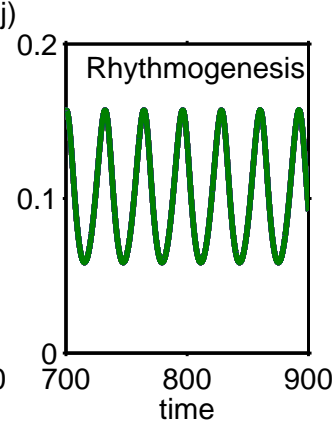

FIG. 9. (Color online) In a network of 16 patches, spatial dynamics and time series: (a, f) for synchronized oscillations for $\epsilon=0.15, d=0.35$ and $m=0.2,(\mathrm{~b}, \mathrm{~g})$ synchronized oscillations with inhomogeneous limit cycles for $\epsilon=0.2369, d=0.35$ and $m=0.2,(\mathrm{c}, \mathrm{h})$ oscillation death for $\epsilon=0.2058, d=0.35$ and $m=0.2,(\mathrm{~d}, \mathrm{i})$ amplitude death for $\epsilon=0.55, d=0.1$ and $m=0.2$, and $(\mathrm{e}, \mathrm{j})$ rhythmogenesis for $\epsilon=0.15, d=0.3$ and $m=0.3$. Other fixed parameters are $\alpha=1, r=0.5, K=0.5, B=0.16$, $\beta=0.5, r_{1}=0.8, K_{1}=2, C=1$ and $\gamma=0.6$.

that consumer species in 16 patches are in-phase synchronized. As like two patches, network of 16 patches also show AD and OD. Depending on initial conditions, we get either IHLC or oscillation death. Spatiotemporal dynamics and time series of multi-clustered OD states are shown in Figs. 9(c) and 9(h), respectively.

For low dispersal rate $(d=0.1), \mathrm{AD}$ occurs. The suppression of oscillation in homogeneous steady states is shown in Fig. 9(d) with the time series shown in Fig. 9(i). Occurrence of rhythmogeneis is shown in Fig. 9(e) and 9(j) for $m=0.3, \epsilon=0.15$ and $d=0.3$. The environmentally coupled system of a network shows similar kind of dynamics shown for 2 patches. From the collective behavior shown in Fig. 9, it is clear that HLC, IHLC, AD, OD and rhythmogeneis all are also valid in a network connected by common dynamic environment.

\section{DISCUSSION}

In summary, in this paper we have modeled an ecological system connecting local habitats through dispersal in a common environment in which the considered environment is nonlinear and dynamic; at the same time we also consider the interaction between the patches and the common dynamic environment is essentially nonlinear, which is controlled by ecologically relevant parameters like conversion efficiency and half saturation con- stant. We highlight that the consumer interactions in three distinct ways such as the interaction within the patch, between the patch and through the environment, however all are interrelated in the collective behavior once coupling involves in the system. Specifically, our study reveals the role of environmental coupling and the effects of inter-patch dispersal on a spatially extended Rosenzweig-MacArthur model using various bifurcation techniques. With simple diffusive coupling in species dispersal, the coupled system exhibits many interesting dynamics with variability in spatial and environmental parameters. Starting from generation of oscillations, various synchronization processes in a smooth oscillator and its suppression to different steady states such as $\mathrm{AD}$, OD and $\mathrm{AD}-\mathrm{OD}$ transition are shown in an ecological system. From different steady states and oscillation, the relationship between synchrony and stability induced by dispersal as well as induced by the dynamic environment has been identified. Even though resource density is not directly involved in dispersal, the intrinsic dynamics and the consumer-resource interaction within the patch enables same qualitative behavior in all the species involved in system. Further, the environmental correlation due to species dispersal enables the persistence of the ecosystem via stabilization of oscillating populations.

Earlier, landscape and metapopulation models have been used to predict the species extinction risk and spatial pattern on ecological processes [18, 34, 35]. Although 
our model depicts as metapopulation model, but explicitly describes various ecological perspectives qualitatively and quantitatively. In particular, dispersal enhances the occurrences of synchronized oscillations and synchronystability relationship. In biological system functioning, synchronization of rhythms plays important role [36, 37]. Rhythmogenesis is an emergent behavior of dynamical system since restoration of oscillations can be achieved easily even if the system is in a steady state. Also, due to the presence of noise in ecological systems, oscillating species are prone to extinction easily, so it is better to understand the factors which enhance the synchronystability relationship. Although many internal and external factors influence in dispersal for successful colonization in the new habitat, but different environmental conditions due to heterogeneity enable the appearance and disappearance of oscillations and transition of homogeneous steady states to inhomogeneous steady states in this system. In general, spatial and environmental heterogeneity clearly distinguish the synchrony-stability relationship induced by both dispersal and a common dynamic environment.

Further, in the context of food web dynamics, food web complexity and species movement pattern contribute largely to enrich the current knowledge [38]. Generally, active as well as passive dispersal take place in natural systems. Specifically, in active dispersal, species are directly involved in movement whereas in passive dispersal, species are being moved by other factors. In this work, we set active dispersal in consumer populations only, but in addition, dispersal also happens in resource populations either directly or indirectly [38, 39]. Further, time scale of dispersal is slow as compare to temporal dynamics within the patch and interconnected habitats are heterogeneous with various network structure. Thus, further study is required to focus on different kind of dispersal with different time scales in heterogeneous environments.

\section{ACKNOWLEDGMENTS}

P.S.D. acknowledges financial support from SERB, Department of Science and Technology (DST), India [Grant No.: YSS/2014/000057]. T.B. acknowledges the financial support from SERB, Department of Science and Technology (DST), Govt. of India [Grant No.: SB/FTP/PS$005 / 2013]$.
[1] I. Hanski, Nature 396, 41 (1998).

[2] I. Hanski, Metapopulation Ecology (Oxford University Press, USA, 1999).

[3] E. E. Goldwyn and A. Hastings, Theoretical Population Biology 73, 395 (2008).

[4] R. Nathan, Proceedings of the National Academy of Sciences USA 105, 19050 (2008).

[5] E. I. Damschen, L. A. Brudvig, N. M. Haddad, D. J. Levey, J. L. Orrock, and J. J. Tewksbury, Proceedings of the National Academy of Sciences USA 105, 19078 (2008).

[6] J. K. Cooper, J. Li, and D. J. S. Montagnes, Ecology Letters 15, 856 (2012).

[7] I. Hanski, Oikos 87, 209 (1999).

[8] D. A. Keith, H. R. Akcakaya, W. Thuiller, G. F. Midgley, R. G. Pearson, S. J. Phillips, H. M. Regan, M. B. Araujo, and T. G. Rebelo, Biology Letters 4, 560 (2008).

[9] T. Banerjee, P. S. Dutta, and A. Gupta, Physical Review E 91, 052919 (2015).

[10] R. Arumugam, P. S. Dutta, and T. Banerjee, Chaos 25, 103121 (2015).

[11] J. Vandermeer, BioScience 56, 967 (2006).

[12] J. E. Keymer, P. A. Marquet, J. X. Velasco-Hernandez, and S. A. Levin, The American Naturalist 156, 478 (2000).

[13] G. Katriel, Physica D: Nonlinear Phenomena 237, 2933 (2008).

[14] V. Resmi, G. Ambika, and R. E. Amritkar, Physical Review E 81, 046216 (2010).

[15] V. Resmi, G. Ambika, and R. E. Amritkar, Physical Review E 84, 046212 (2011).

[16] V. Resmi, G. Ambika, R. E. Amritkar, and G. Rangarajan, Physical Review E 81, 046211 (2012).
[17] D. Ghosh and T. Banerjee, Physical Review E 90, 062908 (2014).

[18] W. Gu, R. Heikkilä, and I. Hanski, Landscape Ecology 17, 699 (2002).

[19] T. Mueller, R. B. O'Hara, S. J. Converse, R. P. Urbanek, and W. F. Fagan, Science 341, 999 (2013).

[20] S. H. Strogatz, Nonlinear Dynamics and Chaos, 1st ed. (Addison-Wesley Publishing Company, Reading, Massachusetts, 1994).

[21] W. W. Murdoch, C. J. Briggs, and R. M. Nisbet, Consumer-Resource Dynamics (Princeton University Press, 2003).

[22] M. Dasgupta, M. Rivera, and P. Parmananda, Chaos 20, 023126 (2010).

[23] A. Chakraborty, A. Ray, S. Basak, and A. R. Chowdhury, Physics Letters A 379, 1418 (2015).

[24] L. Glass, Nature 410, 277 (2001).

[25] W. Zou, D. V. Senthilkumar, N. Nagao, I. Z. Kiss, Y. Tang, A. Koseska, J. Duan, and J. Kurths, Nature Communications 6 (2015).

[26] Y. Loewenstein, Y. Yarom, and H. Sompolinsky, Proceedings of the National Academy of Sciences USA 98, 8095 (2001).

[27] R. M. Harris-Warrick, Progress in Brain Research 187, 213 (2010).

[28] A. Koseska, E. Volkov, and J. Kurths, Physics Reports 531, 173 (2013).

[29] A. Koseska, E. Volkov, and J. Kurths, Physical Review Letters 111, 024103 (2013).

[30] P. S. Dutta and T. Banerjee, Physical Review E 92, 042919 (2015).

[31] M. L. Rosenzweig and R. H. MacArthur, The American Naturalist 97, 209 (1963). 
[32] C. S. Holling, Canadian Entomologist 91, 385 (1959).

[33] B. Ermentrout, Simulating, Analyzing, and Animating Dynamical Systems: A Guide to Xppaut for Researchers and Students (Software, Environments, Tools) (SIAM Press, 2002).

[34] H. R. Akcakaya, V. C. Radeloff, D. J. Mladenoff, and H. S. He, Conservation Biology 18, 526 (2004).

[35] M. C. Wimberly, Landscape Ecology 21, 35 (2006).

[36] L. Glass and M. C. Mackey, From Clocks to Chaos: The Rhythms of Life (Princeton Univ. Press, Princeton,
1988).

[37] A. Goldbeter, Biochemical Oscillations and Cellular Rhythms: The Molecular Bases of Periodic and Chaotic Behaviour (Cambridge Univ. Press, Cambridge, 1996).

[38] P. Amarasekare, Annual Review of Ecology, Evolution, and Systematics 39, 479 (2008).

[39] A. Liebhold, W. D. Koenig, and O. N. Bjørnstad, Annual Review of Ecology, Evolution, and Systematics 35, 467 (2004). 